\title{
Microencapsulated sodium butyrate administered to patients with diverticulosis decreases incidence of diverticulitis - a prospective randomized study
}

\author{
Lukasz Krokowicz • Zoran Stojcev • Bartosz Filip Kaczmarek • \\ Wojciech Kociemba • Elżbieta Kaczmarek • Jaroslaw Walkowiak • \\ Piotr Krokowicz • Michal Drews • Tomasz Banasiewicz
}

Accepted: 20 November 2013 / Published online: 18 December 2013

(C) The Author(s) 2013. This article is published with open access at Springerlink.com

\begin{abstract}
Background Microencapsulated sodium butyrate (MSB) has been previously associated with anti-inflammatory and regenerative properties regarding large bowel mucosa. We aimed to examine a role of MSB in patients with diverticulosis, hypothesizing its potential for reduction of diverticulitis episodes and diverticulitis prevention.

Methods Seventy-three patients with diverticulosis (diagnosed in colonoscopy or/and barium enema or/and CT colography) were recruited for the study and randomized. The investigated group was administered MSB $300 \mathrm{mg}$ daily; the control group was administered placebo. After 12 months, a total of 52 patients completed the study and were subject to analysis (30 subjects and 22 controls). During the study,
\end{abstract}

What is new in this paper? There is limited data about pharmacological prevention of the diverticulitis and complication of the diverticulitis. The prospective randomized study showed that microencapsulated sodium butyrate administered to patients with diverticulosis decreases incidence of diverticulitis. Microencapsulated sodium butyrate is safe and well accepted.

L. Krokowicz $(\bowtie) \cdot$ M. Drews $\cdot$ T. Banasiewicz

Department of General Surgery, Oncologic Gastroenterological

and Plastic Surgery, Poznań University of Medical Sciences,

ul. Przybyszewskiego 49, 60-355 Poznań, Poland

e-mail: 1krokowicz@gmail.com

\section{Z. Stojcev}

Department of General, Vascular and Oncologic Surgery,

Regional Hospital, Słupsk, Poland

\section{Z. Stojcev}

Department of Oncologic Surgery, Medical University,

Gdańsk, Poland

\section{B. F. Kaczmarek}

Department of Urology, Holy Family Hospital, SPZOZ Nad Matką i

Dzieckiem, Poznań, Poland the number of episodes of diverticulitis (symptomatic diagnosis with acute pain, fever, and leukocytosis), hospitalizations, and surgery performed for diverticulitis were recorded. Additionally, a question regarding subjective improvement of symptoms reflected changes in quality of life during the analysis.

Results After 12 months, the study group noted a significantly decreased number of diverticulitis episodes in comparison to the control group. The subjective quality of life in the study group was higher than in the control group. There were no side effects of the MSB during the therapy.

Conclusions MSB reduces the frequency of diverticulitis episodes, is safe, and improves the quality of life. It can play a role in the prevention of diverticulitis.

W. Kociemba

Department of Neuroradiology, Poznań University of Medical

Sciences, Poznań, Poland

E. Kaczmarek

Department of Bioinformatics and Computational Biology,

Poznań University of Medical Sciences, Poznań, Poland

J. Walkowiak

Department of Gastroenterology and Metabolism, Poznań University of Medical Sciences, Poznań, Poland

P. Krokowicz

Department of General and Colorectal Surgery, Poznań University of Medical Sciences, Poznań, Poland 
Keywords Sodium butyrate $\cdot$ Diverticulitis $\cdot$ Diverticulosis . Prevention

\section{Introduction}

Approximately $60 \%$ of individuals over age of 60 living in industrialized countries will develop colonic diverticula [1]. Symptomatic disease is likely to occur in 10 to $25 \%$ of that population [2]. Complications of the diverticulitis can be serious and life-threatening including bowel perforation, abscess, fistula, bleeding, and stricture leading to obstruction. Surgical intervention may be warranted and can range from endoscopic or percutaneous procedures to laparoscopic and open surgery. In the USA alone, complications of diverticulitis are estimated for about 130,000 hospitalizations annually [3]. Developing an effective method of prevention of symptomatic diverticulitis in patient with diverticulosis could lead to a significant reduction in both morbidity and mortality.

The aim of our study was to analyze the preventive effect of microencapsulated sodium butyrate (MSB) in patients with diverticulosis in a prospective randomized trial. We used a single closed-end question to analyze for subjective symptom improvement.

\section{Material and methods}

\section{Study enrollment criteria}

Patients qualified for the study reported diverticulosis diagnosed with colonoscopy, barium enema, and/or abdominal CT no earlier than 1 year before the study, with at least one episode of mild or moderate diverticulitis requiring treatment. The exclusion criteria included age $<18$ years old, previous abdominal surgery (except appendectomy and hernia repair), history of IBD, history of cancer, pharmacotherapy with antibiotics or probiotics within 4 weeks prior to the study, active psychiatric disease, severe comorbidity, pregnancy and breast-feeding, or abdominal symptoms during the last 3 months. In order to avoid bias by dietary changes or tertiary pharmacological agents, the patients were asked not to implement any dietary changes to their typical nutritional habits or use any additional pharmacological treatment throughout the study period.

Patient population and study design

There were a total of 92 patients recruited to the study. Patients were recruited from two coloproctology outpatient clinics in Poznań, Poland. The study was conducted between June 2011 and November 2012 and was designed as a parallel, doubleblinded, randomized, placebo-controlled, per-protocol clinical study, in accordance with the Helsinki Declaration and the regulations of the Ethics Committee of Poznan University of Medical Sciences (26/11) [4]. The study was registered in the EudraCT Trial System with the number 2007-005024-32. During initial visit, patients were informed about the aim of the study and screened for exclusion criteria. Clinical examination was performed and included abdominal examination, body temperature measurement, and WBC count for all patients. At this stage, patients with acute left lower quadrant pain, tenderness, fever $\left(>38{ }^{\circ} \mathrm{C}\right)$, or leukocytosis $\left(\mathrm{WBC}>12,000 / \mathrm{mm}^{3}\right)$ were excluded from the study. After this review, 19 patients were excluded from the study, mainly because of the lack of acceptance, planned surgery, or medical therapy in the next 12 months. The remaining total of 73 patients were randomized to a treatment group $(n=37)$ that received MSB (Debutir ${ }^{\circledR}$, Polfa Łódź) in the dose of $300 \mathrm{mg}$ per day $(2 \times 150 \mathrm{mg})$ for 12 months and to a placebo group $(n=36)$ obtaining identical capsule with placebo at identical regimen also for 12 months. Randomization was done based on the numbers of the patient history (odd numbers, control group; even numbers, treatment group). During the study period, patients were advised to report to the outpatient clinic with abdominal pain, fever, or other symptoms associated with inflammation of the diverticula. The diagnosis criteria of the diverticulitis were defined as acute left lower quadrant pain, tenderness, fever $\left(>38^{\circ} \mathrm{C}\right)$, or leukocytosis $\left(\mathrm{WBC}>12,000 / \mathrm{mm}^{3}\right)$. In case of mild symptoms of diverticulitis, dietary restrictions with rifaximin and probiotics were implemented. In case of moderate or severe symptoms, abdominal imaging was performed: ultrasound for outpatient visits with assessment diverticulitis grade, as proposed by Mizuki et al. [5], and CT of the abdomen and pelvis following Ambrosetti's criteria [6] in hospitalized patients, in case of substantial ultrasound findings and following inability to obtain conclusive ultrasound images (obesity, intestinal gas). Appropriate surgical intervention was to be performed in most severely affected patients after obtaining a CT scan.

Ultrasound and CT scans were done by the same qualified radiologist, with 15 years of experience in ultrasound (US) and CT of the GI tract. Investigation was done as a blinded study — radiologist had no information about patient randomization (placebo or butyrate group).

The study concluded with a final follow-up appointment after 12 months from its initiation. Fifty-two patients reported for the final appointment and completed the study, 30 in the study group and 22 in the placebo group. The main reasons for withdrawal from the study were as follows: lack of interest from the patients, absence during control examinations without reason (14 patients), other diseases without correlation with the gastrointestinal tract (4 patients), moving abroad and lost contact with the patient ( 2 patients), and car accident and serious injury (1 patient). The course of the study is shown in Fig. 1. 
Fig. 1 Flowchart representing the course double-blinded randomized study

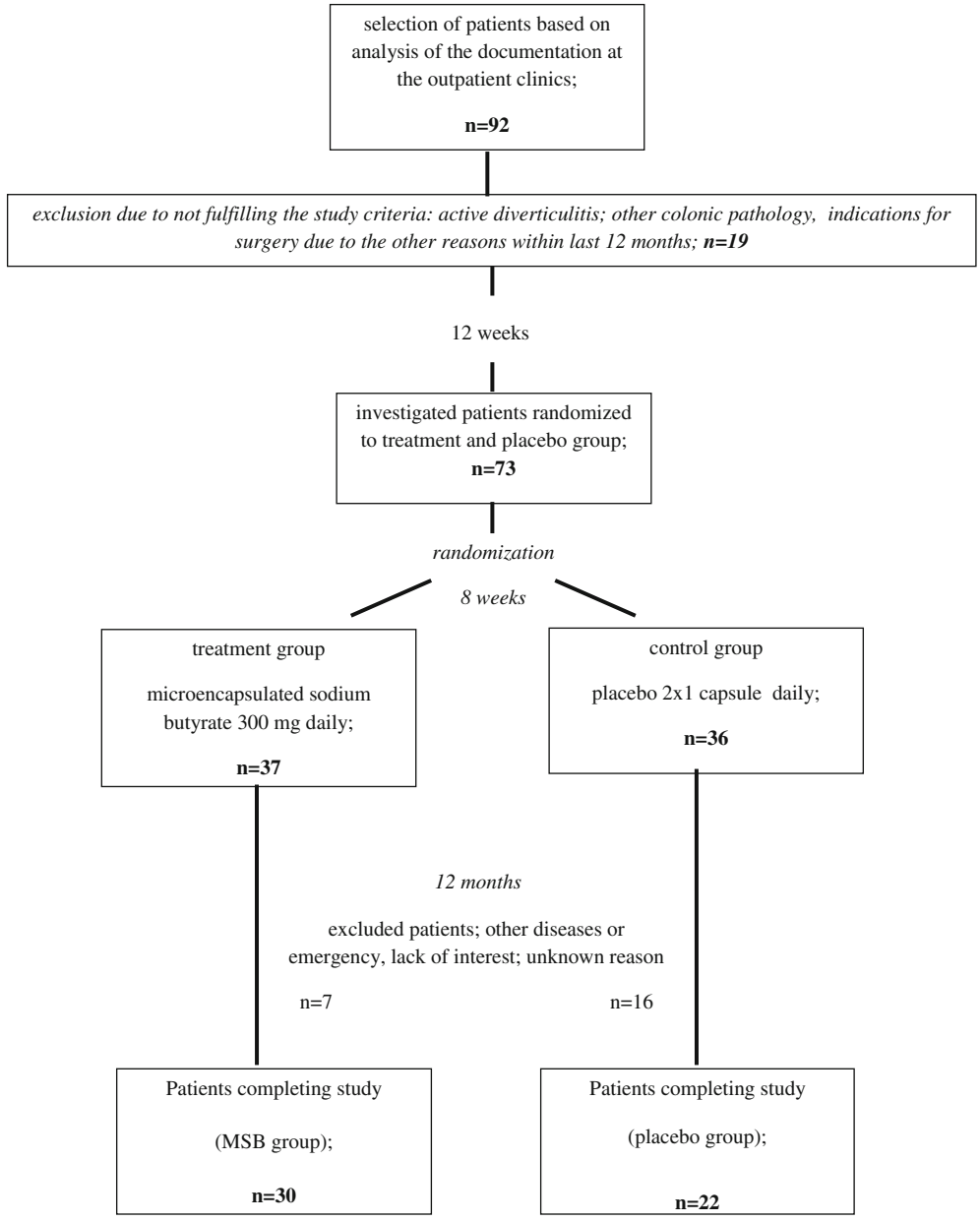

\section{Evaluation of results}

The primary outcome was the number of episodes of diverticulitis experienced by the patients and diverticulitis diagnosed in imaging studies, number of patients hospitalized, and number of patients undergoing surgical intervention for diverticulitis.

The secondary outcome was assessment of subjective symptom improvement with a single closed-end question requiring a "yes" or "no" answer: "Did you observe adequate relief of diverticulosis related to abdominal pain or discomfort within the past 12 months?"

\section{Statistical analysis}

Statistical analysis included medians and quartiles (Q25 and Q75) measuring the degree of dispersion, functioning as median deviation. Additionally, mean values and standard deviations were calculated. The differences between the groups were analyzed with the nonparametric Mann-Whitney test and Kruskal-Wallis test with the Dunn test (post hoc test). Correlation was considered significant at $p<0.05$.

\section{Results}

Between 2011 and 2012, 92 patients were enrolled in the study. After exclusion of 19 patients who did not meet the criteria of the study, 73 patients were randomized into the treatment group or placebo group. After 12 months, a total of 52 patients concluded the study and their results were analyzed. Baseline demographic characteristics of the investigated patient groups as well as detailed results of our study are presented in Table 1.

Mean age of patients in the treatment group $(n=30)$ was $63.7(\mathrm{SD} \pm 6.09)$ and $64.7( \pm 7.65)$ in the placebo group $(n=22)$. There were more females recruited in the study as well as more females randomized in both arms of the study. Duration of diverticular disease in the treatment group was on average 10.47 years $( \pm 4.18)$ and 9.32 years $( \pm 4.82)$ in the placebo group.

The number of diverticulitis clinical symptom occurrences during 12 months of observation was $2(6.67 \%)$ in the MSB group and was significantly lower $(p=0.0425)$ than the clinical symptoms of diverticulitis reported in the control group ( 7 occurrences, $31.8 \%$ ). In comparison to the placebo 
Table 1 Characteristics of the investigated groups (10 years if diverticulosis and longer) and results of the investigation at 12 months of follow-up

\begin{tabular}{|c|c|c|c|c|}
\hline & All patients & Treatment group & Placebo group & $p$ value \\
\hline Number of patients & 52 & 30 & 22 & \multirow{5}{*}{ ns } \\
\hline Gender (female/male) & $30 / 22$ & $17 / 13$ & $13 / 9$ & \\
\hline Mean age of patients (years) & 64.2 & 63.7 & 64.7 & \\
\hline SD & 6.7 & 6.09 & 7.65 & \\
\hline Median & 63.5 & 63 & 64 & \\
\hline Duration of diverticulosis (years) & 9.9 & 10.47 & 9.32 & \multirow[t]{3}{*}{ ns } \\
\hline $\mathrm{SD}$ & 4.46 & 4.18 & 4.82 & \\
\hline Median & 10 & 8.5 & 10 & \\
\hline $\begin{array}{l}\text { Clinical symptoms of diverticulitis: acute left lower quadrant pain, tenderness, } \\
\text { fever }\left(>3{ }^{\circ} 8 \mathrm{C}\right) \text { or leukocytosis }\left(\mathrm{WBC}>12,000 / \mathrm{mm}^{3}\right), n(\%)\end{array}$ & $9(17.3)$ & $2(6.7)$ & $7(31.8)$ & 0.0425 \\
\hline Abdominal ultrasound examinations for suspected diverticulitis, $n(\%)$ & $6(11.5)$ & $1(3.3)$ & $5(22.7)$ & \multirow[t]{2}{*}{0.0229} \\
\hline Diagnoses of diverticulitis in abdominal US, $n(\%)$ & $6(11.5)$ & $1(3.3)$ & $5(22.7)$ & \\
\hline CT abdomen for suspected diverticulitis, $n(\%)$ & $3(5.8)$ & $1(3.3)$ & $2(9)$ & \multirow{2}{*}{$\begin{array}{l}\text { ns (low number } \\
\text { of events) }\end{array}$} \\
\hline Diagnoses of diverticulitis in CT abdomen, $n(\%)$ & $3(5.8)$ & $1(3.3)$ & $2(9)$ & \\
\hline Hospitalizations for diverticulitis, $n(\%)$ & $4(7.7)$ & $1(3.3)$ & $3(13.6)$ & $\begin{array}{l}\text { ns (low number } \\
\text { of events) }\end{array}$ \\
\hline Surgery (Hartmann's procedure) because of diverticulitis, $n(\%)$ & $1(1.9)$ & $0(0)$ & $1(4.5)$ & $\begin{array}{l}\text { ns (low number } \\
\text { of events) }\end{array}$ \\
\hline $\begin{array}{l}\text { Subjective evaluation of the patients (positive answer to the question: Did you } \\
\text { observe adequate relief of diverticulosis related to abdominal pain or discomfort } \\
\text { within the past } 12 \text { months?), } n(\%)\end{array}$ & $22(42.3)$ & $17(56.7)$ & $5(22.7)$ & 0.0143 \\
\hline Number of adverse events, $n(\%)$ & $0(0)$ & $0(0)$ & $0(0)$ & $\begin{array}{l}\text { ns (low number } \\
\text { of events) }\end{array}$ \\
\hline
\end{tabular}

$n s$ not significant

group, the number of abdominal ultrasound examinations performed for suspected diverticulitis and subsequent number of diverticulitis diagnosed based on abdominal US was significantly lower ( 1 vs $5, p=0.0229$ ). The number of abdominal CT imaging performed for suspected diverticulitis, number of hospitalizations for diverticulitis, and number surgical intervention for diverticulitis were reported to be higher in the treatment group ( 1 vs 2,1 vs 3 , and 0 vs 1 , respectively) but were too low to adequately compare the investigated groups.

Subjective improvement in observed symptoms based on a single closed-end question (yes or no answer): "Did you observe adequate relief of diverticulosis related to abdominal pain or discomfort within the past 12 months?" was reported to be higher in the treatment group (55.67 vs $22.73 \%, p=0.0143$ ).

At the time of the study as well as after the conclusion of the study, there were no adverse or side effects of MSB or placebo observed.

\section{Discussion}

In the present study, we sought to determine the protective effect of MSB on incidence of symptomatic diverticulitis in patients with diverticulosis. To determine MSB efficacy, we designed a randomized placebo-controlled study recording rates of clinical symptoms related to diverticulitis as well as patients' subjective impression of symptom improvement over the 12 months. As far as we are aware, our study is the first to assess the value of MSB in the reduction of symptomatic diverticulitis occurrence.

Diverticulitis is not only responsible for increasingly common cause of hospital admissions [7] but also poses a risk of continued episodes of diverticulitis in patients' lifetime. It has been estimated that within 5 years after a successful nonsurgical treatment, up to $80 \%$ diverticulitis can reoccur, leading to impaired quality of life and physical activity with increased costs due to multiple specialist consultations, pain medication, and work absence [8-11].

In order to reduce incidence and recurrence, various methods of diverticulitis prevention have been proposed. Appropriate diet changes including high-vegetable and high-fiber intake and has been proven effective in previous studies $[12,13]$. The protective effect of dietary fiber has been explained by increased bulk of stool, distention of colonic lumen, and a decline in intraluminal pressures, thus reducing colonic transit time [14, 15]. In patients with already developed diverticular disease and patients who are symptomatic, evidence of benefit of increased fiber intake seems less established. Apart from dietary changes, reduced body mass as well as an inverse association between vigorous physical activity and occurrence of diverticular disease has been demonstrated [16]. 
With regard to pharmacological approach, an emergent role of cyclic nonabsorbable antibiotic has been extensively investigated. Increased efficacy of rifaximin in diverticular disease symptom reduction when compared to simple dietary fiber supplementation has been established [17]. A study by Tursi et al. has demonstrated a significant advantage of combined efficacy of rifaximin plus mesalazine against rifaximin alone in observed severity of symptoms, improved bowel habits, and diverticulitis recurrence [18].

Administration of probiotics is known to reduce incidence of inflammatory disease of the bowel and has been associated with benefit in symptomatic diverticulosis [19-21]. The probiotics act by influencing antimicrobial agent production and interact competitively for metabolites with proinflammatory organisms. The combination of Lactobacilli spp. with rifaximin seems effective in the reduction of severe diverticulitis and can prevent recurrences, thus minimizing the need for surgical treatment [20].

In the current study regarding diverticulitis occurrence reduction, we utilized MSB, a short-chain fatty acid previously proven to provide symptomatic relief in patients suffering from various range of colonic diseases, such as IBS, inflammatory bowel disease, diarrhea, and malabsorption and suggestive of preventive role in cancerogenesis of colonocytes [22-24]. While there is no certainty as to what renders sodium butyrate (SB) to be so beneficial in such a wide range of colonic diseases, it has been established that SB can act as a regulator of intestinal environment. It is a preferred energy substrate for colonocytes, can moderate intestinal permeability, reduce oxidative stress, and reinforce colonic defense barrier leading to decreased inflammation of mucosa, increased cell regeneration rate, and promote healing [25-27]. What prompted the authors to study the efficacy of SB for diverticulitis was the lack of side effects of SB $[28,29]$ and similarities between diverticulitis and irritable bowel syndrome for which SB has been shown effective, such as presence of abnormal colonic motility, visceral hypersensitivity, presence of low-grade inflammation, and increased circulating levels of either substance $\mathrm{P}$ or vasoactive intestinal polypeptide [30, 31].

We utilized a microencapsulated form of SB to maximize the biological effect on the colon. Unprotected forms of SB induce fast absorption in the small bowel, thus preventing its passage to the large intestine where local release of SB seems to be of most benefit. In our study, we utilized a unique lipid membrane microcapsulation, designed to release SB distal from the ileo-cecal region and used successfully in previous studies [22]. Other formulas to release SB in the colon have been used, including hydroxylpropyl methylcellulose and Shellac coating [32].

Several disease-specific questionnaires based on the clinical symptoms are used to evaluate GI symptoms in patients with diverticulitis [33]. These can be time-consuming, which can be a limitation in an efficiency-oriented clinical practice. There are questionnaires based on a single closed-end question providing an efficient measurement of subjective colonic symptom improvement, previously validated for other diseases such as IBS. The validated IBS questionnaire utilizes a question: "Did you observe adequate relief of irritable bowel syndrome symptoms related to abdominal pain or discomfort within the past week?" [34]. Although the result is not qualitative, it seems to adequately reflect the efficacy of treatment as seen by patients. Prior to our study, we were seeking for a simplified assessment of symptom improvement for diverticulitis. Based on IBS symptom improvement question, and taken similar symptoms related to disease, we established a modified question for symptom improvement related to diverticulitis. The relief of symptoms was investigated using a single closed-end question: "Did you observe adequate relief of diverticulosis related to abdominal pain or discomfort within the past 12 months?" In comparison to the placebo group, we recorded improved relief in the studied group within the treatment group. In our opinion, it is a very important assessment tool of the implemented therapy that reflects its combined efficacy. This type of validation can be useful in daily clinical practice to simplify the validation procedure. This instrument is a validated and generally accepted simple outcome assessment of IBS treatment efficacy $[34,35]$. We are, of course, aware of the large simplification methods for assessing performance through lack of validated life quality questionnaire.

Overall, our findings demonstrate that diverticulosis patients administered MSB regimen suffer from a smaller number of episodes typical of diverticulitis than the placebo group $(6.67 \%$ vs $31.8 \%, p=0.0425)$. We postulate that this reflects the preventive properties of MSB in this group of patients. With regard to a single closed-end question for symptom assessment, we recorded subjective improvement in comparison to the placebo group (56.67 vs $22.73 \%$, $p=0.0143$ ). This can further implicate increased quality of life in patients with diverticulosis upon MSB regimen. From practical standpoint, this could translate into future implementation of MSB in patients with diverticulosis. In smaller scale, preventive therapy could be applied alone or with other agents for groups at higher risk of recurrence, which have previously been identified and include patients with family history of disease, long segment of diseased colon, and history of retroperitoneal abscess at first presentation [24].

Current analysis has several limitations. Increased power of study would be beneficial, especially in the context of events that were not frequent and did not reach enough power to attain statistical significance. Diagnosis criteria of diverticulitis are based on clinical symptoms which are subjective to the physician's individual assessment and experience, thus making standardization and comparison 
difficult. Although the question for subjective clinical symptom improvement seemed practical, it has not been validated for diverticular disease. Lastly, the authors feel that a larger study with multi-institutional input would be valuable.

\section{Conclusions}

Administration of MSB in asymptomatic patients with diverticulosis can lead to significant decrease of clinical diverticulitis incidence $(p=0.425)$. In addition, it can decrease the need for imaging studies associated with diverticulitis $(p=0.0229)$. After a 12-month course of MSB in patients with diagnosed diverticulosis, a subjective improvement in occurrence of clinical symptoms associated with diverticular disease was reported $(p=0.0143)$. No adverse effects associated with MSB administration have been observed. Management of diverticulosis with MSB is safe and can play an important role in the prevention of progression to clinical diverticulitis.

Conflict of interest The following authors have no conflict of interest to disclose: Krokowicz Lukasz, Stojcev Zoran, Kaczmarek Bartosz Filip, Kociemba Wojciech, Kaczmarek Elżbieta, Walkowiak Jarosław, Krokowicz Piotr, Drews Michał, and Banasiewicz Tomasz.

Open Access This article is distributed under the terms of the Creative Commons Attribution License which permits any use, distribution, and reproduction in any medium, provided the original author(s) and the source are credited.

\section{References}

1. Parks TG (1975) Natural history of diverticular disease of the colon. Clin Gastroenterol 4:3-21

2. Floch MH, Bina I (2004) The natural history of diverticulitis: fact and theory. J Clin Gastroenterol 38:S2-S7

3. Munson KD, Hensien MA, Jacob LN, Robinson AM, Liston WA (1996) Diverticulitis: a comprehensive follow-up. Dis Colon Rectum 39:318-324

4. World Medical Association (2008) WMA Declaration of Helsinkiethical principles for medical research involving human subjects, Seoul, Korea

5. Mizuki A, Nagata H, Tatemichi M, Kaneda S, Tsukada N, Ishii H, Hibi $\mathrm{T}$ (2005) The out-patient management of patients with acute mild-tomoderate colonic diverticulitis. Aliment Pharmacol Ther 21:889-897

6. Ambrosetti P, Jenny A, Becker C, Terrier TF, Morel P (2000) Acute left colonic diverticulitis - compared performance of computed tomography and water-soluble contrast enema: prospective evaluation of 420 patients. Dis Colon Rectum 43(10):1363-1367

7. Etzioni DA, Mack TM, Beart RW Jr, Kaiser AM (2009) Diverticulitis in the United States: 1998-2005: changing patterns of disease and treatment. Ann Surg 249:210-217

8. Krukowski ZH, Matheson NA (1984) Emergency surgery for diverticular disease complicated by generalized and faecal peritonitis: a review. Br J Surg 71:921-927

9. Wong WD, Wexner SD, Lowry A, Vernava A III, Burnstein M (2000) Practice parameters for the treatment of sigmoid diverticulitis- supporting documentation. The Standards Task Force. The American Society of Colon and Rectal Surgeons. Dis Colon Rectum 43:290-297

10. Parks TG (1969) Natural history of diverticular disease of the colon. A review of 521 cases. Br Med J 4:639-642

11. Peppas G, Bliziotis LA, Oikonomaki D, Falagas ME (2007) Outcomes after medical and surgical treatment of diverticulitis: a systematic review of the available evidence. J Gastroenterol Hepatol 22:1360-1368

12. Brodribb AJ (1977) Treatment of symptomatic diverticular disease with a high-fibre diet. Lancet 1:664-666

13. Ornstein MH, Littlewood ER, Baird IM, Fowler J, North WR, Cox AG (1981) Are fibre supplements really necessary in diverticular disease of the colon? A controlled clinical trial. Br Med J (Clin Res Ed) 282: $1353-1356$

14. Lupton JR, Turner ND (1999) Potential protective mechanisms of wheat bran fiber. Am J Med 106:24-27

15. Tan KY, Seow-Choen F (2007) Fiber and colorectal diseases: separating fact from fiction. World J Gastroenterol 13:4161-4167

16. Gronek P, Holdys J. (2013) Genes and physical fitness. Trends in Sport Sciences 20(1):16-29.

17. Tursi A, Brandimarte G, Daffinà R (2002) Long-term treatment with mesalazine and rifaximin versus rifaximin alone for patients with recurrent attacks of acute diverticulitis of colon. Dig Liver Dis 34:510-515

18. Colecchia A, Vestito A, Pasqui F, Mazzella G, Roda E, Pistoia F (2007) Efficacy of long term cyclic administration of the poorly absorbed antibiotic rifaximin in symptomatic, uncomplicated colonic diverticular disease. World J Gastroenterol 13:264-269

19. Fric P, Zavoral M (2003) The effect of non-pathogenic Escherichia coli in symptomatic uncomplicated diverticular disease of the colon. Eur J Gastroenterol Hepatol 15:313-315

20. Giaccari S, Tronci S, Falconieri M, Ferrieri A (1993) Long-term treatment with rifaximin and lactobacilli in post-diverticulitic stenoses of the colon. Riv Eur Sci Med Farmacol 15:29-34

21. Lamiki P, Tsuchiya J, Pathak S, Okura R, Solimene U, Jain S (2010) Probiotics in diverticular disease of the colon: an open label study. J Gastrointestin Liver Dis 19:31-36

22. Banasiewicz T, Krokowicz L, Stojcev Z, Kaczmarek BF, Kaczmarek E, Maik J, Marciniak R, Krokowicz P, Walkowiak J, Drews M (2013) Microencapsulated sodium butyrate reduces the frequency of abdominal pain in patients with irritable bowel syndrome. Colorectal Dis 15: 204-209

23. Cordel S, Dupas B, Douillard JY, Meflah K (1998) Interleukin-2/ sodium butyrate treatment cures rats bearing liver tumors after acquired 5-fluorouracil resistance. Int J Cancer 78:735-739

24. Hall JF, Roberts PL, Ricciardi R (2011) Long-term follow-up after an initial episode of diverticulitis: what are the predictors of recurrence? Dis Colon Rectum 54:283-288

25. Vernia $P$, Annese V, Bresci G, d'Albasio G, D'Incà R, Giaccari S (2003) Topical butyrate improves efficacy of 5-ASA in refractory distal ulcerative colitis: results of a multicentre trial. Eur J Clin Investig 33:244-248

26. Hamer HM, Jonkers D, Venema K, Vanhoutvin S, Troost FJ, Brummer RJ (2008) Review article: the role of butyrate on colonic function. Aliment Pharmacol Ther 27:104-119

27. Topping DL, Clifton PM (2001) Short-chain fatty acids and human colonic function: roles of resistant starch and nonstarch polysaccharides. Physiol Rev 81:1031-1064

28. Canani RB, Terrin G, Cirillo P, Castaldo G, Salvatore F, Cardillo G, Coruzzo A, Troncone R (2004) Butyrate as an effective treatment of congenital chloride diarrhea. Gastroenterology 127:630-634

29. Schmitt MG Jr, Soergel KH, Wood CM (1976) Absorption of short chain fatty acids from the human jejunum. Gastroenterology 70: 211-215

30. Clemens CH, Samsom M, Roelofs J, van Berge Henegouwen GP, Smout AJ (2004) Colorectal visceral perception in diverticular disease. Gut 53:717-722 
31. Petruzziello L, Iacopini F, Bulajic M, Shah S, Costamanga G (2006) Review article: uncomplicated diverticular disease of the colon. Aliment Pharmacol Ther 23:1379-1391

32. Roda A, Simoni P, Magliulo M, Nanni P, Baraldini M, Roda G, Roda E (2007) A new oral formulation for the release of sodium butyrate in the ileo-cecal region and colon. World $\mathrm{J}$ Gastroenterol 13:1079-1084

33. Zullo A, Hassan C, Maconi G, Manes G, Tammaro G, De Francesco V, Annibale B, Ficano L, Buri L, Gatto G, Lorenzetti R, Campo SM, Ierardi
E, Pace F, Morini S (2010) Cyclic antibiotic therapy for diverticular disease: a critical reappraisal. J Gastrointestin Liver Dis 19:295-302

34. Bijkerk CJ, de Wit NJ, Muris JW, Jones RH, Knottnerus JA, Hoes AW (2003) Outcome measures in irritable bowel syndrome: comparison of psychometric and methodological characteristics. Am J Gastroenterol 98:122-127

35. Irvine EJ, Whitehead WE, Chey WD, Matsueda K, Shaw M, Talley NJ et al (2006) Design of treatment trials for functional gastrointestinal disorders. Gastroenterology 130:1538-1551 